\title{
OTIMIZAÇÃO OPERACIONAL DE UM REATOR CATALÍTICO VIA PROGRAMAÇÃO NÃO-LINEAR
}

\author{
A. P. MELO, N. S. V. BARBOSA, E. Q. EUGENIO e A. L. H. COSTA \\ Universidade do Estado do Rio de Janeiro, Instituto de Química \\ E-mail para contato: alice_peccini@yahoo.com.br
}

\begin{abstract}
RESUMO - Reatores químicos podem ser considerados o coração de uma planta de processo, onde a matéria-prima é convertida no produto de interesse comercial. Por esta razão, a adequada operação de um reator é fundamental para rentabilidade do processo. Em função desta importância, o presente trabalho apresenta a aplicação de ferramentas de programação matemática na forma de um problema de programação não-linear, visando maximizar o lucro operacional. O sistema estudado corresponde a um reator catalítico modelado através de equações de balanço clássicas. Na formulação do problema de otimização, as restrições são basicamente representadas por um conjunto de equações oriundas dos balanços discretizados através da técnica de diferenças finitas. A função objetivo é representada pela receita total associada à corrente de saída. A aplicação da metodologia proposta em um exemplo típico da prática industrial indica um ganho financeiro relevante em relação a estratégias operacionais convencionais.
\end{abstract}

\section{INTRODUÇÃO}

Uma vez que o processo de transformação das matérias-primas em produtos ocorre nos reatores, estes são muitas vezes considerados o coração da planta de processo (Towler e Sinnott, 2013). No decorrer do tempo de atividade de uma planta de processos industriais, a otimização se torna fundamental a fim de promover um aumento da receita e menores custos. O cenário mundial atual é um cenário competitivo, onde as empresas e indústrias buscam elevado desempenho, eficiência e retorno financeiro (Fuad et al., 2012).

No contexto da engenharia de processos, destacam-se dois tipos de otimização: de projeto e operacional. A otimização operacional de uma planta de processos pode ser aplicada em uma gama de variáveis. Devido à existência de um conjunto de fatores a serem considerados no processo de otimização, um modelo que aborde a performance de um reator com relação às variáveis de entrada é necessário para maximizar o índice econômico (Zhou e Yuan, 2005). Exemplos de trabalhos anteriores aplicados à otimização operacional de reatores químicos podem ser encontrados em Lovik et al. (1998) e Zahedi et al.(2005)

O presente trabalho objetiva a formulação e implementação de uma programação matemática na forma de um problema de programação não-linear, visando maximizar a produção de um determinado produto de interesse. O sistema estudado corresponde a um reator catalítico isotérmico ao longo da sua extensão modelado através de equações de balanço clássicas. Na formulação do problema de otimização, as restrições são basicamente representadas por um conjunto de equações oriundas dos balanços discretizados através da técnica de diferenças finitas. 


\section{MODELAGEM DO SISTEMA}

O sistema estudado é composto por um reator catalítico de leito fixo (Packed-Bed Reactor - PBR), onde ocorre uma rede de reações, em fase gasosa. O reator é tubular, de diâmetro, comprimento, pressão de operação, vazão e composição das correntes de entrara conhecidos. Considera-se, por hipótese, que os sistemas de troca térmica presentes sejam capazes de garantir uma condição de temperatura constante ao longo do comprimento do reator. A queda de pressão é considerada desprezível ao longo do reator.

Uma vez que a maioria dos catalisadores não mantém suas atividades nos mesmos níveis por períodos indefinidos, é necessário avaliar a taxa de desativação para planejar a operação do reator de forma eficiente. O decaimento da atividade ao longo do tempo afeta significativamente a produção de uma planta de processos e pode ser acelerado pelo aumento da temperatura.

Para a modelagem do sistema, aplicou-se um balanço material por componente em um determinado volume de controle do reator, obtendo-se:

$$
\frac{d F_{i}}{d z}=a \rho_{B} A_{T} \sum_{j}\left[M_{i, j} F A_{j} \exp \left(\frac{-E_{j}}{T_{t e}}\right) \prod_{i \in L_{j}}\left(\hat{p}_{i}{ }^{-M_{i, j}}\right)\right]=f_{i}(z)
$$

em que $F_{i}$ é a vazão molar da espécie $i$ em $\mathrm{kmol} / \mathrm{s}, z$ é o comprimento do reator em $\mathrm{m}, a$ é a atividade do catalisador, $M_{i, j}$ é o coeficiente estequiométrico da espécie $i$ na reação $j, F A_{j}$ é o fator pré-exponencial da reação $j$ em kmol/(kg.s.bar), $E_{j}$ é a razão entre a energia de ativação da reação $j$ pela constante universal dos gases em $\mathrm{K}, T$ é a temperatura do sistema em $\mathrm{K}, \hat{p}_{i}$ é a pressão parcial do componente $i, \rho_{B}$ é a massa específica do leito em $\mathrm{kg} / \mathrm{m}^{3}$ e $A_{T}$ é a área transversal do leito em $\mathrm{m}^{2}$ e $L_{j}$ é o conjunto das espécies $i$ onde $M_{i, j}<0$.

A pressão parcial é dada por:

$$
\frac{\hat{p}_{i}}{P}=y_{i}=\frac{F_{i}}{\sum_{i} F_{i}}
$$

sendo $P$ a pressão de operação do reator e $y_{i}$ a fração molar do componente $i$. O modelo de desativação do catalisador baseou-se em Lovik et al. (1998):

$$
\frac{d a}{d t}=-K_{c a t} \exp \left[-E_{c a t}\left(\frac{1}{T_{t e}}-\frac{1}{T_{0}}\right)\right] a^{5}=g(t)
$$

em que $K_{c a t}$ é a constante de desativação $\mathrm{em} \mathrm{h}^{-1}, E_{c a t}$ é razão entre a energia de ativação do decaimento pela constante universal dos gases em $\mathrm{K}$ e $T_{0}$ a temperatura de referência em $\mathrm{K}$.

Na modelagem acima considera-se que a desativação é lenta, o que permite descrever o comportamento do reator como um sucessão de estados estacionários (abordagem pseudoestacionária). 


\section{FORMULAÇÃO DO PROBLEMA DE OTIMIZAÇÃO}

A seguir, são apresentados os índices, variáveis, restrições e função objetivo utilizados na formulação do problema.

\subsection{Indexação e Variáveis}

Os índices utilizados para identificar as variáveis e equações do problema são $i \in$ I para as espécies, $j \in \mathrm{J}$ para as reações, $n \in \mathrm{N}$ para os intervalos de comprimento do reator na discretização e te $\in \mathrm{T}$ para os intervalos de tempo de operação.

As variáveis de decisão do problema são as temperaturas do reator em cada tempo, que são determinadas de acordo com a solução do problema de otimização. As outras variáveis envolvidas são: a atividade do catalisador, as vazões, frações molares e pressões parciais de cada espécie $i$ em cada ponto do reator e em cada tempo. Estas variáveis são determinadas de acordo com as restrições provenientes do modelo do sistema.

\subsection{Função Objetivo}

A função objetivo consiste na maximização da produção de $\mathrm{B}$ ao longo do tempo de operação da planta, e logo tem-se:

$$
\text { Função Objetivo }=\sum_{t e} w_{t e} M_{B, t e}
$$

sendo $M_{B, t e}$ a vazão mássica do componente B na saída do reator em ton/h no tempo te e $w_{t e}$ refere-se aos pesos do método de integração de Simpson $1 / 3$.

\subsection{Restrições}

Para a implementação do problema no GAMS é necessário transformar as equações diferenciais provenientes da modelagem em equações algébricas, o que foi realizado através da técnica numérica de Runge-Kutta de segunda ordem, aplicado às equações 1 e 3, obtendose:

$$
\begin{aligned}
& F_{i, n+1, t e}=F_{i, n, t e}+\frac{h}{2}\left[f_{i, n, t e}+f_{i, n+1, t e}\right] \quad \forall i \in \mathrm{I}, t e \in \mathrm{T}, n \in \mathrm{N}^{*} \\
& a_{n, t e+1}=a_{n, t e}+\frac{h^{\prime}}{2}\left[g_{n, t e}+g_{n, t e+1}\right] \quad \forall n \in \mathrm{N}, t e \in \mathrm{T}^{*}
\end{aligned}
$$

sendo $h$ o passo relativo ao comprimento do reator e $h^{\prime}$ o passo referente ao tempo. $\mathrm{O}$ sobrescrito * indica a exclusão do último elemento do conjunto.

A Equação 2 relativa a relação entre as frações e vazões molares se torna a seguinte restrição: 


$$
\hat{p}_{i, n, t e} \sum_{i} F_{i, n, t e}=F_{i, n, t e} P \quad \forall i \in \mathrm{I}, t e \in \mathrm{T}, n \in \mathrm{N}
$$

Buscando facilitar a convergência, foram inseridas restrições que limitam a variação da temperatura entre instantes vizinhos:

$$
T_{t e+1}-\delta \leq T_{t e} \leq T_{t e+1}+\delta \quad \forall t e \in \mathrm{T},
$$

Finalmente, a otimização se baseia na maximização da Equação 4, restrita às Equações 5 a 8, complementadas por equações associadas à limites mínimos e máximos para as variáveis.

\section{RESULTADOS}

\subsection{Dados do problema}

A aplicação da abordagem proposta é ilustrada por um exemplo composto de seis reações com 4 espécies envolvidas (Figura 1).

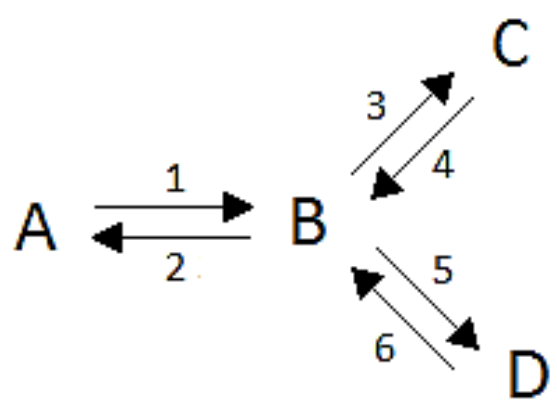

Figura 1 - Representação da rede reacional

A corrente de entrada consiste em $10 \mathrm{kmol} / \mathrm{s}$ de A. O produto de interesse, cuja vazão se deseja maximizar, é o produto intermediário B. Considerou-se que as reações apresentam cinética de primeira ordem, cujos parâmetros estão representados na Tabela 1.

\begin{tabular}{|c|c|c|c|c|c|c|}
\hline Reação & 1 & 2 & 3 & 4 & 5 & 6 \\
\hline $\begin{array}{l}\text { Fator pré-exponencial } \\
\left(\mathrm{kmol} \mathrm{kg}^{-1} \cdot \mathrm{s}^{-1} \cdot \mathrm{bar}^{-1}\right)\end{array}$ & $259,2 \mathrm{E}-6$ & $21,6 \mathrm{E}-6$ & $43,2 \mathrm{E}-6$ & $216 \mathrm{E}-6$ & $64,8 \mathrm{E}-6$ & $324 \mathrm{E}-6$ \\
\hline Energia de ativação (K) & 1200 & 750 & 2000 & 1500 & 3000 & 2500 \\
\hline
\end{tabular}

Tabela 1 - Condições experimentais

Os índices aplicáveis ao problema, assim como os parâmetros necessários para a resolução do mesmo encontram-se nas Tabelas 2 e 3, respectivamente. 
Tabela 2- Indexação do sistema

\begin{tabular}{ccc}
\hline Índice & \multicolumn{2}{c}{ Referência } \\
\hline $\mathbf{i}$ & Componente & $\mathrm{A}, \mathrm{B}, \mathrm{C}$ e D \\
$\mathbf{j}$ & Reação & 1 a 6 \\
$\mathbf{n}$ & Posição no reator & 0 a 35 \\
te & Intervalo de tempo & 0 a 36 \\
\hline
\end{tabular}

Tabela 3- Parâmetros do sistema

\begin{tabular}{cc}
\hline Parâmetro & Valor \\
\hline Pressão de operação $(\mathbf{b a r})$ & 60 \\
Massa específica do leito $\left(\mathbf{k g} / \mathbf{m}^{\mathbf{3}}\right)$ & 1154 \\
Diâmetro do reator $(\mathbf{m})$ & 3 \\
Comprimento do reator $(\mathbf{m})$ & 3,5 \\
Atividade inicial do catalisador & 1 \\
Constante de desativação $\left(\mathbf{h}^{-\mathbf{1}}\right)$ & $5 \times 10^{-7}$ \\
Energia de ativação do decaimento $(\mathbf{K})$ & 10000 \\
Temperatura de referência $(\mathbf{K})$ & 413 \\
Tempo de operação da planta $(\mathbf{a n o})$ & 3 \\
Passo da integração de Simpson $(\mathbf{h})$ & 730 \\
Massa Molar do produto de interesse $(\mathbf{k g} / \mathbf{k m o l})$ & 32 \\
\hline
\end{tabular}

\subsection{Solução do Problema}

A formulação do problema de otimização proposto foi resolvida utilizando o software GAMS através do solver CONOPT. A dimensão do problema alcança um total de 30748 variáveis.

A maximização da função objetivo resultou em uma vazão mássica média de 888,7 ton/h. Para fins comparativos, estipulou-se dois diferentes cenários onde a otimização não teria sido aplicada, um no qual a desativação do catalisador não seria prevista na operação do reator catalítico, que trabalharia a temperatura constante de $250^{\circ} \mathrm{C}$. O segundo onde a desativação do catalisador tivesse sido considerada no estabelecimento da política operacional do reator ao longo da campanha, através da operação via uma rampa de temperatura, variando de 170 a $300^{\circ} \mathrm{C}$. Os perfis de temperatura, atividade e vazão mássica ao longo do tempo nos diferentes cenários podem ser observados nas Figuras 2, 3 e 4, respectivamente. 


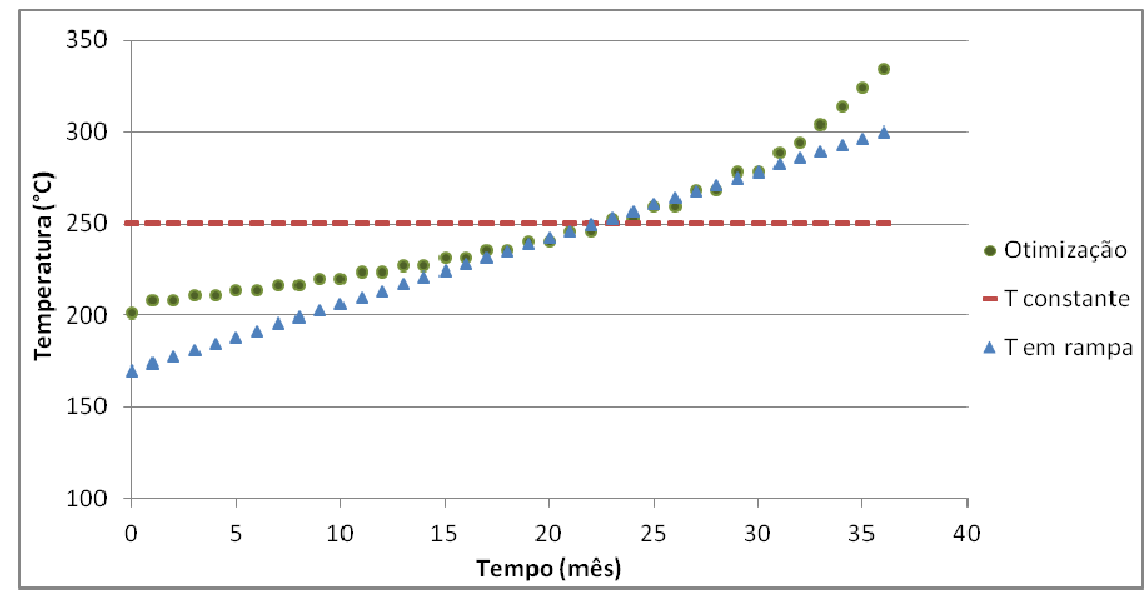

Figura 2 - Perfil de temperatura ao longo do tempo nos diferentes cenários analisados

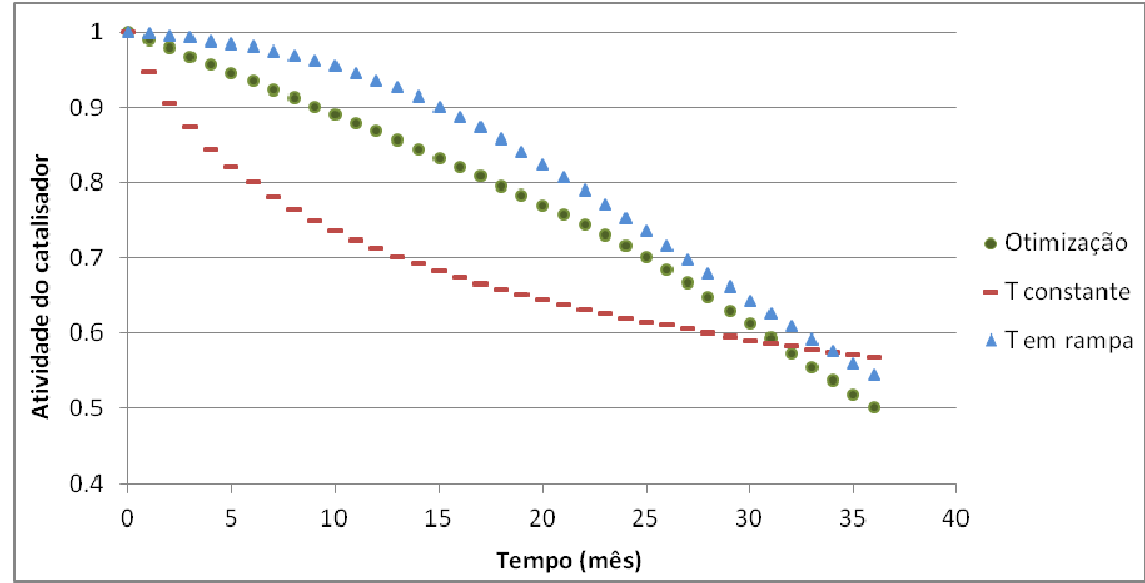

Figura 3 - Perfil da atividade do catalisador ao longo do tempo nos diferentes cenários analisados

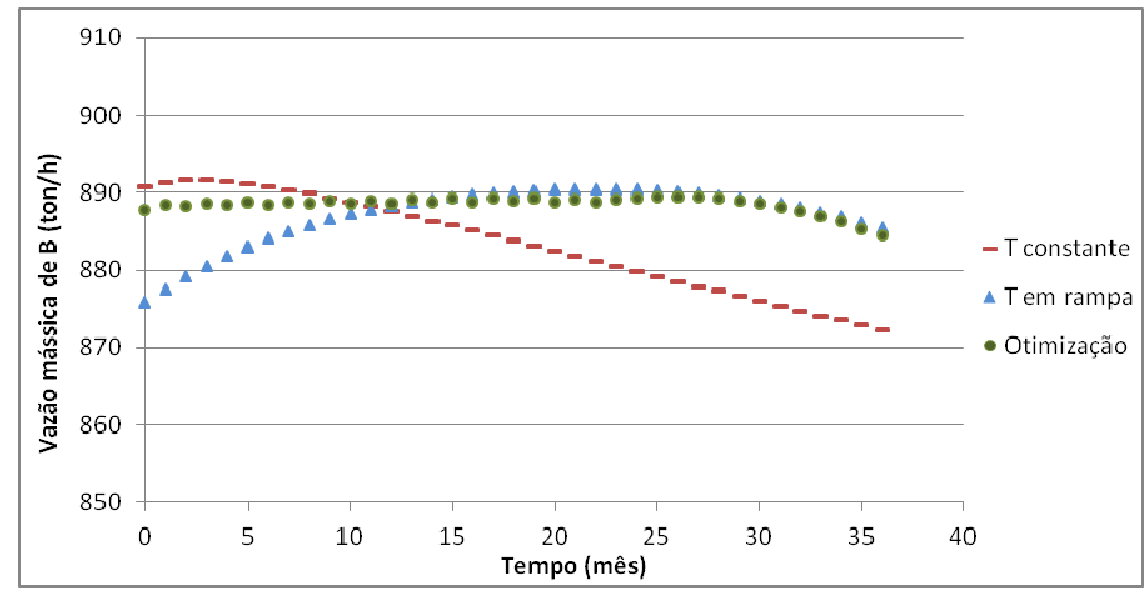

Figura 4 - Perfil de vazão mássica do produto de interesse nos diferentes cenários analisados 
Pode-se observar na Figura 2 que o perfil de temperatura da situação otimizada apresenta caráter ascendente a fim de compensar a desativação do catalisador. Analisando a influência da temperatura, verifica-se que o aumento desta favorece o aumento da taxa reacional do produto de interesse, porém é necessário encontrar um equilíbrio uma vez que o produto de interesse é o intermediário da rede reacional. Além disso, o aumento da temperatura acelera a desativação do catalisador.

Analisando as figuras anteriores e comparando os casos com temperatura otimizada e temperatura em rampa, verifica-se que no início da operação, a temperatura otimizada apresenta maiores valores. Este aumento da temperatura inicial promove uma desativação mais acelerada, como pode ser observado na Figura 3. Porém, conforme mostrado na Figura 4, a vazão mássica do produto de interesse é mais elevada nesta situação, o que justifica o perfil de otimização.

Comparando os perfis relativos à situação com temperatura otimizada e temperatura constante, verifica-se que a temperatura constante inicia-se em um platô mais elevado (Figura 2), o que promove uma maior vazão mássica nos períodos iniciais de operação (Figura 4). Por outro lado, tal fato ocasiona a desativação mais acelerada do catalisador (Figura 3) gerando o decaimento acelerado da vazão mássica nos períodos posteriores.

$\mathrm{Na}$ Tabela 4, são apresentados os resultados de produção do componente de interesse nos três casos, evidenciando um maior ganho alcançado através da otimização. Apesar destas variações serem relativamente pequenas em termos percentuais, em termos absolutos, estas podem implicar em benefícios financeiros consideráveis. Por exemplo, tomando por base o preço de B de US\$ 514,82 por tonelada, típico de uma commodity química (e.g. metanol) são explicitados na Tabela 4, o ganho alcançado pela otimização frente às políticas convencionais de operação.

Tabela 4 - Comparativos da produção e receita do produto de interesse

\begin{tabular}{ccc}
\hline Cenário & Produção de B (ton/ano) & Ganho (milhões US\$/ano) \\
\hline Otimização & 7785216 & - \\
Temperatura Constante & 7738368 & 24 \\
Temperatura em Rampa & 7772928 & 6 \\
\hline
\end{tabular}

\section{CONCLUSÕES}

O presente trabalho apresentou a formulação de um problema de programação matemática para a otimização dinâmica da operação de um reator catalítico associada a qualquer rede de reações. Através do emprego de técnicas de discretização, as equações diferenciais foram transformadas em um conjunto de relações algébricas, resultando em um problema de programação não linear (NLP). Através de um exemplo envolvendo quatro componentes, os resultados alcançados pela técnica proposta trouxeram ganhos financeiros consideráveis quando comparados às políticas operacionais convencionais, sem a utilização de uma ferramenta de otimização. 


\section{REFERÊNCIAS}

FUAD, M. N. M.; HUSSAIN, M. A.; ZAKARIA, A. Optimization strategy for long-term catalyst deactivation in a fixed-bed reactor for methanol synthesis process. Comput. Chem. Eng., v. 44, p. 104-126, 2012.

LOVIK, I.; HILLESTAD, M.; HETZBERG, T. Long term dynamics optimization of a catalytic reactor system. Comput. Chem. Eng., v. 22, suppl., p. 707-710, 1998.

TOWLER, G.; SINNOTT, R. K. Design of Reactors and Mixers. In: Chemical Engineering Design. 2. ed. Oxford: Elsevier, 2013. cap. 15, p. 631-751.

ZAHEDI, G, ELKAMEL, A., LOHI, A., Dynamic Optimization Strategies of a Heterogeneous Reactor for CO2 Conversion to Methanol, Energy \& Fuels, v. 21, p. 2977-2983, 2007.

ZHOU, X.; YUAN, W. Optimization of the fixed-bed reactor for ethylene epoxidation. Chem. Eng. Process.,v. 44, n. 10, p. 1098-1107, 2005. 